Галочкин В. И.

\title{
ПЕРЕЧИСЛЕНИЕ РЕШАЮЩИХ ДЕРЕВЬЕВ ОГРАНИЧЕННОЙ СТОИМОСТИ НА И-ИЛИ ДЕРЕВЕ
}

\begin{abstract}
Аннотация: Рассматриваются И-ИЛИ деревья с заданной стоимостью дуг либо вершин, широко применяемые в системах искусственного интеллекта. Описывается алгоритм типа ветвей и грании, позволяющий перечислить все решающие деревья, стоимость которыхне превышает наперед заданной константы. Трудоемкость получения очередного решающего дерева составляет $\mathrm{O}(\mathrm{N})$, где $N$ - количество вершин И-ИЛИ дерева. Указывается способ стековой организации информации, позволяющий свести затраты памяти к величине $\mathrm{O}(\mathrm{N})$ без изменения прежней оценки трудоемкости. Выполнена программная реализация описанного алгоритма, подтвердившая при тестировании полученные теоретические оценки трудоемкости и объема необходимой памяти. Эффективность поиска повышена благодаря введенному понятию минимальной оболочки И-ИЛИ дерева по ограничению стоимости, что позволяет гарантировать наличие допустимых решающих деревьев при спуске по дереву решений. Решающие поддеревья перечисляются не по отдельности, а блоками в виде поддеревьев И-ИЛИ дерева, в которых все варианты допустимы. Ключевые слова: Искусственный интеллект, Алгоритм, Перечисление, И-ИЛИ граф, И-ИЛИ дерево, Решающее дерево, Дерево решения, Вариант И-ИЛИ дерева, Стоимость, Ограничение по стоимости
\end{abstract}

\section{Введение}

И-ИЛИ деревья широко применяются в системах искусственного интеллекта [1-4]. На основе И-ИЛИ деревьев строятся, например, методы сведения задач к подзадачам [1, 3] и способы описания множества технических решений определенного класса [2].

И-ИЛИ дерево содержит два типа вершин: И-вершины и ИЛИ-вершины. Решающим деревом (деревом решения) [1] называется такое поддерево, которое строится сверху вниз и включает корень, всех сыновей для каждой своей И-вершины и единственного сына для каждой ИлИ-вершины. Будем для краткости называть решающее дерево вариантом, как это сделано в [4].

Распространенной задачей для И-ИЛИ деревьев является перечисление вариантов с определенными свойствами [1-4]. В [1] рассматривается задача выбора варианта минимальной стоимости. Стоимость варианта определяется суммарной стоимостью его дуг. В [2] количественные показатели приписываются вершинам И-ИЛИ дерева, а не дугам. Мы будем следовать такому представлению. Фактически, оно получается простым переносом 
стоимости дуги на вершину, в которую она направлена. При обратном преобразовании стоимость вершины переносится на стоимость дуги, ведущей в данную вершину.

Рассмотрим следующую задачу. В вершинах И-ИЛИ дерева определены значения стоимости. Стоимостью варианта считается суммарная стоимость его вершин. Требуется перечислить все варианты И-ИЛИ дерева, для которых стоимость не превышает заданной константы $C$.

\section{1. Минимальная оболочка}

Итак, имеется И-ИЛИ дерево $D$ с $N$ вершинами. Обозначим заданное значение стоимости в вершине $i$ через $w_{i}$. Функция стоимости $W$ определена рекурсивно на множестве вершин варианта путем обхода дерева варианта от листьев к корню. Действительно, для вершины $z$ с сыновьями $z_{i}$ при $i=1,2, \ldots, k$ и стоимостью $w_{z}$

$$
W(z)=w_{z}+W\left(z_{1}\right)+W\left(z_{2}\right)+\ldots+W\left(z_{k}\right) .
$$

Стоимостью всего варианта является стоимость его корня.

Назовем поддеревом вариантов множество всех вариантов такого поддерева, которое включает корень, всех сыновей для каждой своей И-вершины и не менее одного сына для каждой ИЛИ-вершины. Пересечение поддеревьев вариантов представляет собой множество вариантов подобного И-ИЛИ поддерева, поэтому также является поддеревом вариантов.

Будем считать допустимыми варианты И-ИЛИ дерева, удовлетворяющие ограничению стоимости $W \leq C$. Назовем минимальной оболочкой И-ИЛИ дерева по ограничению $W \leq C$ такое поддерево вариантов, которое

- включает все допустимые варианты исходного И-ИЛИ-дерева;

- $\quad$ не может быть усечено без потери допустимых вариантов.

Из этого определения следует, что каждая вершина минимальной оболочки входит в какой-либо допустимый вариант.

Рассмотрим способ построения минимальной оболочки. Для любой вершины $z$ найдем минимальный отрезок $\left[a_{z}, b_{z}\right]$, в котором лежат все возможные значения стоимости. Будем называть $\left[a_{z}, b_{z}\right]$ отрезком возможных значений стоимости в вершине $z$.

Выполним обход дерева в направлении от листьев к корню $r$.

Пусть $z_{i}(i=1,2, \ldots, k)$ - сыновья вершины $z, w_{z}$ - заданная стоимость вершины $z$, a $\left[a_{i}, b_{i}\right]$ - рассчитанные отрезки возможных значений стоимости в вершинах $z_{i}$ (для листьев $\left.a_{i}=b_{i}=z_{i}\right)$.

Если $z-$ И-вершина, то $a_{z}=w_{z}+a_{1}+a_{2}+\ldots+a_{k} ; b_{z}=w_{z}+b_{1}+b_{2}+\ldots+b_{k}$.

Если $z-$ ИЛИ-вершина, то $a_{z}=w_{z}+\min \left(a_{i}\right) ; b_{z}=w_{z}+\max \left(b_{i}\right)$ для $i=1,2, \ldots, k$.

Таким образом находятся отрезки возможных значений для всех вершин И-ИЛИ дерева. Если окажется, что в корне $a_{r}>C$, то минимальная оболочка пуста. Это означает, что допустимые варианты отсутствуют. Если же $b_{r} \leq C$, то любой вариант И-ИЛИ дерева 
является допустимым, а минимальная оболочка совпадает со всем деревом. Будем далее считать, что $a_{r} \leq C \leq b_{r}$, что гарантирует наличие допустимых вариантов.

Следующий обход выполняется наоборот от корня дерева к листьям. При тех же обозначениях для любой вершины $z_{i}$ найдем максимальное значение стоимости $h_{i}$, при котором может существовать допустимый вариант, включающий вершину $z_{i}$.

Пусть для вершины $z$ со стоимостью $w_{z}$ и сыновьями $z_{i}(i=1,2, \ldots, k)$ указанное значение равно $h_{z}$ (для корня $h_{r}=C$ ).

Если $z-$ И-вершина, рассмотрим для каждого $i$ уравнение

$$
w_{z}+a_{1}+a_{2}+\ldots+a_{i-1}+h_{i}+a_{i+1}+\ldots+a_{k}=h_{z},
$$

откуда

$$
h_{i}=h_{z}-\left(w_{z}+a_{1}+a_{2}+\ldots+a_{i-1}+a_{i+1}+\ldots+a_{k}\right) .
$$

Если $z-$ ИЛИ-вершина, то отсечем те вершины $z_{i}$ для которых $w_{z}+a_{i}>h_{z}$. Для остальных вершин $h_{i}=h_{z}-w_{z}$.

Легко видеть, что в результате описанной процедуры получается поддерево решений $T$, являющееся минимальной оболочкой дерева $D$ по ограничению $W \leq C$. Трудоемкость построения минимальной оболочки оценивается величиной $O(N)$.

\section{2. Допустимые поддеревья вариантов}

Будем называть допустимым такое поддерево вариантов, в котором каждый вариант является допустимым. Допустимые поддеревья вариантов можно выделять, распространяя последовательно от корня к листьям ограничения на стоимость.

Пусть для вершины z со стоимостью $\mathrm{w}_{z}$ и сыновьями $z_{i}(i=1,2, \ldots, k)$ получено ограничение $W(z) \leq d_{z}$ (для корня $d_{r}=C$ ). Наша задача получить ограничения вида $W\left(z_{i}\right) \leq d_{i}$

Если $z$ - И-вершина, подберем произвольные значения $a_{i} \leq d_{i} \leq b_{i}$ такие, что

$$
w_{z}+d_{1}+d_{2}+\ldots+d_{k}=d_{z}
$$

и будем считать, что в $i$-й вершине получено ограничение $W\left(z_{i}\right) \leq d_{i}$.

Можно, например, определять величины $d_{i}$ по формулам $d_{i}=d_{i}(t)=a_{i}+t\left(b_{i}-a_{i}\right)$, решая уравнение (1) относительно $t$.

Если $z$ - ИЛИ-вершина, отсечем вершины $z_{i^{\prime}}$ для которых $w_{z}+a_{i}>d_{z^{\prime}}$ а для остальных вершин положим $d_{i}=d_{z}-w_{z}$.

В итоге выделяется допустимое поддерево вариантов $P$. Будем говорить, что $P$ получено в результате спуска ограничения $W \leq C$.

В общем случае, минимальная оболочка не является допустимым поддеревом вариантов, ногарантированно содержитвариантминимальной стоимости.Трудоемкость получения допустимого поддерева вариантов оценивается величиной $O(N)$. 


\section{3. Разложение разности минимальной оболочки и допустимого поддерева}

Теорема. Пусть $T$ - минимальная оболочка И-ИЛИ дерева, а $P$ - допустимое поддерево вариантов, полученное в результате спуска ограничения $W \leq C$, причем $P \neq T$. Тогда существуют такие поддеревья вариантов $S_{i}(i=1,2, \ldots, m ; m \leq N)$, что

1) $T \backslash P=\bigcup_{i=1}^{m} S_{i}$;

2) $S_{i} \cap S_{j}=\varnothing$ при $i \neq j$;

3) каждое поддерево вариантов $S_{i}$ содержит допустимые варианты.

Доказательство. Пусть $X$ - множество вершин из $T, y$ - произвольная ИЛИ-вершина $P$. Введем следующие обозначения:

- $\quad Z(X, T)$ - множество вариантов из $T$, не содержащих вершин множества $X$;

- $G(X, T)$ - множество вариантов из $T$, содержащих хотя бы одну вершину из $X$;

- $\quad R(y, P)$ - множество сыновей ИЛИ-вершины $y \in P$, не лежащих в $P$;

- $\quad H(P, T)$ - множество ИЛИ-вершин $y_{i} \in P$, для которых $R\left(y_{i}, P\right) \neq \varnothing$.

Заметим, что если вершина $z_{i}$ является сыном И-вершины $Z$ и $z_{i} \in X$, то варианты множества $Z(X, T)$ не могут содержать вершину $z$. Очевидно, что $Z(X, T)$ является поддеревом вариантов и для любых множеств вершин $A$ и $B$

$$
Z(A \cup B, T)=Z(A, T) \cap Z(B, T) .
$$

Множество $G(R(y, P), T))$ также является поддеревом вариантов. Легко проверяется равенство

$$
T \backslash P=\bigcup_{y_{i} \in H(P, T)} G\left(R\left(y_{i}, P\right), T\right) .
$$

Положим

$$
S_{i}=G\left(R\left(y_{i}, P\right), T\right) \bigcap Z\left(\bigcup_{j<i} G\left(y_{j}, T\right)\right), i=1,2, \ldots, m .
$$

Из равенства (2) и определения $S_{i}$ следует справедливость первых двух утверждений теоремы. Докажем, что выполняется и третье утверждение.

Зафиксируем номер $i \leq m$. По определению минимальной оболочки, существует допустимый вариант $u \in G\left(R\left(y_{i}, P\right), T\right)$. Выберем все вершины $y_{i_{k}}(k=1,2, \ldots, q)$ такие, что $i_{k}<i$ и $u \in G\left(R\left(y_{i_{k}}, P\right), T\right)$.

Обозначим через $u_{i_{k}}$ поддерево с корнем $y_{i_{k}}$, являющееся частью варианта $u$, а через $t_{i_{k}}$ поддерево с корнем $y_{i_{k}}$, являющееся частью варианта минимальной стоимости.

По построению $P$, поддерево $t_{i_{k}}$ целиком лежит в $P$. Сформируем новый вариант $t$, заменив в варианте $u$ все поддеревья $u_{i_{k}}$ на поддеревья $t_{i_{k}}(k=1,2, \ldots, q)$. Значения 
стоимости $W$ на варианте $t$ не больше, чем на варианте $u$, поэтому $t$ также является допустимым вариантом. Поскольку поддеревья $t_{i_{k}}$ лежат в $P$, то $t \in Z\left(\bigcup_{j<i} G\left(y_{j}, T\right)\right)$. Следовательно, $t \in S_{i^{\prime}}$ и
теорема доказана.

\section{4. Алгоритм перечисления допустимых вариантов}

На основании доказанной теоремы построим алгоритм типа ветвей и границ [5] перечисления допустимых поддеревьев вариантов. Сохраняя обозначения теоремы, выделим из исходного И-ИЛИ дерева $D$ минимальную оболочку $T$, а из $T$ путем спуска ограничения $W \leq C$ найдем допустимое поддерево вариантов $P$.

Если $P \neq T$, определим множество $H(P, T)$, вершину $y_{1} \in H(P, T)$ и поддерево вариантов $\left.S_{1}=G\left(R\left(y_{1}, P\right), T\right)\right)$. По доказанному, в $S_{1}$ имеются допустимые варианты. Выделим из $S_{1}$ минимальную оболочку $T_{1}$ и допустимое поддерево вариантов $P_{1}$.

Как и ранее, если $P_{1} \neq T_{1}$, найдем множество $H\left(P_{1}, T_{1}\right)$, вершину $y_{11} \in H\left(P_{1}, T_{1}\right)$ и поддерево вариантов $S_{11}$ из которого будет выделено очередное допустимое поддерево вариантов $P_{11}$.

Продолжим подобный поиск в глубину, пока очередное допустимое поддерево вариантов не совпадет с минимальной оболочкой. Тогда вернемся к предыдущей минимальной оболочке и выделим на ней следующее допустимое поддерево вариантов. Ввиду того, что минимальная оболочка всегда содержит допустимые варианты, очередное допустимое поддерево вариантов находится с трудоемкостью $O(N)$.

Обозначим через $|A|$ число вершин в поддереве вариантов $A$. Поскольку при любой последовательности номеров $i_{1}, i_{2}, \ldots, i_{k}$ выполняется

$$
T \supset T_{i_{1}} \supset T_{i_{1} i_{2}} \supset \ldots \supset T_{i_{1} i_{2} \ldots i_{k}},
$$

TO

$$
N \geq\left|T_{i_{1}}\right|>\left|T_{i_{1} i_{2}}\right|>\ldots>\left|T_{i_{1} i_{2} \ldots i_{k}}\right|
$$

и цепочка (3) содержит не более $N$ элементов.

Обозначим через $E_{i_{1}}$ множество вершин из $T \backslash T_{i_{1}}$, а через $E_{i_{1} i_{2} \ldots i_{k}}$ при $k>1$ - множество вершин из $T_{i_{1} i_{2} \ldots i_{k-1}} \backslash T_{i_{1} i_{2} \ldots i_{k}}$. При реализации алгоритма можно хранить в памяти минимальную оболочку $T$ и текущую цепочку множеств $E_{i_{1}}, E_{i_{1} i_{2}}, \ldots, E_{i_{1} i_{2} \ldots i_{k}}$. Аналогичным образом сохраняется информация о допустимых поддеревьях вариантов $P, P_{i_{1}}, P_{i_{1} i_{2}}, \ldots, P_{i_{1} i_{2} \ldots i_{k}}$.

После исчерпания допустимых вариантов из $T_{i_{1} i_{2} \ldots i_{k}}$ легко восстановить минимальную оболочку $T_{i_{1} i_{2} \ldots i_{k-1}}$, допустимое поддерево вариантов $P_{i_{1} i_{2} \ldots i_{k-1}}$ и перейти к выделению следующего допустимого поддерева вариантов, обновив необходимую информацию в памяти. Как видно из (4), для этого требуется объем памяти порядка $N$.

Описанный способ организации информации фактически предполагает использование стека. Объем вычислений при этом по-прежнему имеет порядок $N$.

Отметим в заключение, что алгоритм позволяет выбирать допустимые варианты не 
по отдельности, а блоками в виде поддеревьев И-ИЛИ дерева, в которых все варианты допустимы, то есть удовлетворяют ограничению по стоимости $W \leq C$.

\section{Библиография :}

1. Нильсон, Н. Искусственный интеллект. Методы поиска решений / Н. Нильсон. - М.: Мир, 1973. - 270 с.

2. Автоматизация поискового конструирования / А. И. Половинкин, Н. К. Бобков, Г. Я. Буш и др. Под редакцией А. И. Половинкина. - М.: Радио и связь, 1981. -344 с.

3. Братко, И. Программирование на языке Пролог для искусственного интеллекта / И. Братко - М.: Мир, 1990. -560 c.

4. Кручинин, В.В. Методы построения алгоритмов генерации и нумерации комбинаторных объектов на основе деревьев И/ИЛИ / В.В. Кручинин. - Томск: В-Спектр, 2007. - 200 С.

5. Рейнгольд, Э. Н. Комбинаторные алгоритмы. Теория и практика / Э. Рейнгольд, Ю. Нивергельт, Н. Део. - М.: Мир, 1980. -476 с.

\section{References:}

1. Nil'son, N. Iskusstvennyi intellekt. Metody poiska reshenii / N. Nil'son. - M.: Mir, 1973. - 270 s.

2. Avtomatizatsiya poiskovogo konstruirovaniya / A. I. Polovinkin, N. K. Bobkov, G. Ya. Bush i dr. Pod redaktsiei A. I. Polovinkina. - M.: Radio i svyaz', 1981. -344s.

3. Bratko, I. Programmirovanie na yazyke Prolog dlya iskusstvennogo intellekta / I. Bratko - M.: Mir, 1990 - 560 s.

4. Kruchinin, V.V. Metody postroeniya algoritmov generatsii i numeratsii kombinatornykh ob"ektov na osnove derev'ev I/LLI / V.V. Kruchinin. - Tomsk:V-Spektr, 2007. - 200 s.

5. Reingol'd, E. N. Kombinatornye algoritmy. Teoriya i praktika / E. Reingol'd, Yu. Nivergel't, N. Deo. - M.: Mir, 1980. $-476 \mathrm{~s}$. 